\title{
Educación con sentido holístico en el devenir de la Sistemología Interpretativa
}

\section{Education with a holistic sense in the evolution of Interpretive Systemology}

\author{
Ramsés Fuenmayor \\ Centro de Investigaciones en Sistemología Interpretativa \\ Facultad de Ingeniería, Universidad de Los Andes \\ Mérida-Venezuela \\ ramsesfa@gmail.com
}

\begin{abstract}
Resumen-Con el propósito de dar cuenta del Proyecto de Educación de la Sistemología Interpretativa y de su escuela piloto, se presenta una versión resumida del devenir de la Sistemología Interpretativa, pues es a partir de la narrativa de dicho devenir que se podrá mostrar el sentido particular y las características fundamentales de dicho desarrollo educativo. En el relato histórico se pueden distinguir dos etapas: La primera, en la que, por una parte, se construyó una ontoepistemología holística, una teoría de organizaciones de actividades humanas y una teoría del diseño; y, por la otra, se realizó una serie de estudios e intervenciones en organizaciones públicas. En la segunda etapa, como consecuencia de lo encontrado en la primera, se emprendió una investigación histórica-ontológica que permitiera entender la pobreza y fragmentación del sentido de la vida y de lo que en ella ocurre; pobreza y fragmentación que caracterizan el presente de las sociedades occidentales y occidentalizadas. Las preguntas prácticas surgidas de esta investigación histórica-ontológica condujeron a la puesta en práctica de un proyecto de investigación-acción en torno a la posibilidad de una educación escolar caracterizada por la enseñanza-aprendizaje con/en sentido holístico. Se describe brevemente esa experiencia.
\end{abstract}

Keywords- Sistemología Interpretativa, Educación holística, Pensamiento-práctica de sistemas, Onto-epistemología

Abstract-In order to account for the Education Project of the Interpretive Systemology and its pilot school, a summary version of the evolution of Interpretive Systemology is presented. In this historical narrative two stages can be distinguished: The first, in which, on the one hand, a holistic onto-epistemology, a theory of organizations of human activities and a theory of design were constructed; and, on the other, a series of studies and interventions were carried out in various public organizations. In the second stage, as a consequence of what was found in the first, a historical-ontological investigation was undertaken to understand the poverty and fragmentation of the meaning of life and of what occurs in it - poverty and fragmentation that characterize the present of Occidental and Occidentalized societies. The practical questions arising from this historical-ontological investigation led to the implementation of an action-research project concerning an education characterized by teaching-learning with/in a holistic sense. That experience is briefly described.

Keywords- Interpretive Systemology, Holistic Education, Systems Thinking-practice, Onto-epistemology

\section{Introducción ${ }^{1}$}

La Sistemología Interpretativa es un programa de investigación y una disciplina cuyos orígenes datan de

\footnotetext{
${ }^{1}$ La primera parte de este artículo, la referente a la
} narración del devenir de la Sistemología Interpretativa, ha sido tomada, con algunas modificaciones, de mi libro “El Cultivo de la Verdad” (Fuemayor, 2016) hace ya cuatro décadas. En el desarrollo de este programa de investigación podemos distinguir dos etapas: una primera que aproximadamente podría situarse entre el inicio del segundo quinquenio de los años setenta y el fin de la década de los ochenta del Siglo XX; y una segunda etapa que se iniciaría desde el comienzo de los noventa de ese mismo siglo pasado y llegaría hasta nuestros días. 
2. Primera etapa: laconstrucción de una ontoepistemología de sistemas y una teoría de organizaciones sistémico-interpretativa.

La Sistemología Interpretativa -y, por tanto, su primera etapa - surge de la reunión o confluencia de dos interrogantes, inquietudes o problemas académicos:

\subsection{Primer interrogante}

En el segundo quinquenio de los años setenta, los que iniciamos esta disciplina que ahora llamamos Sistemología Interpretativa éramos jóvenes profesores de la Escuela de Ingeniería de Sistemas de la Universidad de Los Andes. En el ambiente internacional, la Ingeniería de Sistemas se perfilaba como una no muy bien definida disciplina y carrera universitaria cuyo propósito parecía ser -así lo entendíamos a partir de nuestras lecturas- el del estudio de situaciones problemáticas complejas ${ }^{2}$ bajo el enfoque de sistemas con una orientación práctica ${ }^{3}$. El enfoque de sistemas, directriz de la joven disciplina, se ofrecía como un modo de estudiar y actuar bajo la premisa "holística” de que el todo trasciende la reunión de las partes. Sin embargo, para aquel entonces, realmente aun no era "un modo de estudiar y actuar" ya establecido; más bien era una intención de desarrollar "un modo de estudiar y actuar bajo la premisa holística”. Dicha intención estaba fundada en un ataque al marcado énfasis reduccionista-analítico de la práctica científica y tecnológica que cada vez se acentuaba más a lo largo del Siglo XX. El argumento anti-reduccionista y antianalítico del enfoque de sistemas se podía resumir en los siguientes términos: “aislar un fenómeno de su contexto (reduccionismo) y comenzar su estudio por una separación en sus partes (análisis a-priori) implica perder de vista la condición holística de cualquier fenómeno”. A raíz de esta intención sistémica comenzaban a surgir métodos sistémicos para el estudio de organizaciones de actividades humanas —objeto predilecto de la nueva disciplina. Sin embargo, asombrosamente, la pregunta básica ante el lema fundamental del enfoque de sistemas quedaba desatendida por los estudiosos del "movimiento de sistemas".

En efecto, preguntas tales como: ¿cómo es posible que el todo trascienda la reunión de sus partes? ¿cómo es posible el conocimiento del todo en cada caso? ¿por qué la práctica científica parece ignorar esa intuición holística básica? eran continuamente evadidas por los precursores y seguidores de la nueva disciplina. Esas preguntas obviamente exigían la formulación de una base teórica para el enfoque de sistemas. Esa base teórica no sería otra cosa que una onto-epistemología que diera cuenta de la condición holística de lo que ocurre y de la posibilidad de su estudio.

\footnotetext{
${ }^{2}$ Las organizaciones de actividades humanas fueron el campo principal de estudio de tales "situaciones problemáticas complejas”.

${ }^{3}$ Cuando decimos que tales estudios tenían una “orientación práctica” queremos decir que pretendían derivar racionalmente cursos de acción a partir del estudio en cuestión.
}

Vimos allí, en el proyecto de construcción de una onto-epistemología para el enfoque de sistemas, una digna y apasionante tarea que podría contribuir considerablemente a consolidar una ciencia de sistemas. Su importancia se hacía más acuciante al constatar que la práctica de la ingeniería y ciencia de sistemas, en nuestra Escuela de Ingeniería de Sistemas y en otras instancias universitarias del mundo, parecía alejarse de aquellos principios que considerábamos originales. En efecto, vimos con preocupación, que la práctica de la ingeniería de sistemas estaba siendo orientada a un propósito muy diferente del de comprender y cuestionar el sentido de organizaciones de actividades humanas de manera de orientarlas hacia el bien común. Por el contrario, la ingeniería y ciencia de sistemas, en su quehacer efectivo, se perfilaba como un poderoso instrumento para diseñar y mantener complejas organizaciones de las que jamás se preguntaría por su sentido o papel social. Teníamos la intuición de que este marcado instrumentalismo, asfixiante en nuestra escuela y en otros muchos nidos de la práctica mundial de sistemas, iba de la mano con el descuido que la joven disciplina mostraba por comprender su propio principio sistémico. Más tarde esta intuición se convertiría en uno de los acicates de la Sistemología Interpretativa.

Y así fue. Más adelante, gracias a lo andado en ese camino de investigación, fuimos comprendiendo que el instrumentalismo, más allá de ser una tendencia, un interés, que se adueñaba de ciertas disciplinas (como paradójicamente ocurría con la ingeniería de sistemas), era un modo de ser cultural que estaba amenazando gravemente la posibilidad de hacer sentido holístico de lo que ocurre. Quiero decir que observamos un deterioro de la posibilidad del sentido holístico, no sólo en la ciencia y en la tecnología, sino en la vida cotidiana de aquellos que vivimos en culturas occidentales y, muy especialmente, de los que vivimos en culturas "occidentalizadas" 4 . Esta observación amplió nuestro foco de interés: desde el que se concentraba en la promoción del estudio del sentido holístico de organizaciones de actividades humanas hasta un interés mucho más general por la recuperación del sentido holístico de la vida cotidiana.

\subsubsection{Las caras inquisitivas y políticas de la primera inquietud.}

Quisiera aquí abrir un paréntesis en esta narración para llamar la atención sobre lo siguiente: La primera inquietud de la Sistemología Interpretativa presentaba dos caras: una inquisitiva y una política. La cara inquisitiva es obvia: queríamos comprender cómo es posible que el todo trascienda a la reunión de las partes y cuáles consecuencias epistemológicas se derivan de esta condición ontológica. Pero, del otro lado de esta

\footnotetext{
${ }^{4}$ Llamo culturas “occidentalizadas” a las que devinieron de la imposición de una cierta forma, generalmente marginal, de la cultura occidental a otra cultura que originalmente era no-occidental. Las instituciones de estas culturas occidentalizadas guardan la apariencia de ser occidentales, aunque en su interior alberguen

importantes contradicciones culturales. Claros ejemplos de culturas occidentalizadas son las sociedades latinoamericanas y buena parte de las sociedades "subdesarrolladas".
} 
cara inquisitiva, menos visible, palpitaba una cara política: estábamos, por una parte, intentando reformar una disciplina - la de sistemas - que intuíamos mal fundamentada y minada por una contradicción fundamental entre su principio holístico y su quehacer instrumental. Pero, por otra parte, habitaba en nosotros el sueño de contribuir con un mundo mejor en el que lo que ocurre pueda tener un sentido más rico y pleno. Digo que era una "cara política” porque de eso trata la política en su forma original y auténtica: de la participación pública de los seres humanos destinada a mejorar sus sociedades. Espero que el lector note cómo, en este caso, ambas caras, la política y la inquisitiva, son “caras de una misma moneda”; una no podría ser sin la otra. Pero, además, es importante notar que el aspecto político implicaba un enfrentamiento contra lo que lucía como dominante: la racionalidad instrumental en la ingeniería de sistemas, en la ciencia y en la tecnología en general, y, más allá y más preocupante: en la vida de las sociedades modernas. Todo esto implicaba un compromiso intelectual y afectivo con nuestra actividad laboral que trascendía con mucho la mera rutina movida por el incentivo de un salario y el temor de perder un cargo.

He aquí la primera inquietud (e interrogante), la cual, reunida con la que ahora pasamos a explicar, constituyeron el umbral del camino de la Sistemología Interpretativa.

\subsection{Segundo interrogante}

Decíamos que el objeto de estudio privilegiado de la nueva disciplina de sistemas eran las organizaciones de actividades humanas (organizaciones tales como las empresas, las instituciones públicas, las organizaciones de acción comunitaria, las cooperativas, etc.). En este campo de las organizaciones de actividades humanas, nos llamaba la atención un fenómeno que difícilmente escapaba a la vista de alguien que con detenimiento y actitud reflexiva observara y pensara en las organizaciones públicas venezolanas; a saber: la gran brecha que existía entre el discurso justificador de estas instituciones y su actual papel social. A esta cualidad general que creíamos encontrar en muchas de las instituciones venezolanas (en los años setenta) la llamamos "esquizofrenia institucional". 5

¿Ocurría realmente este fenómeno de "esquizofrenia institucional” en las instituciones públicas venezolanas? De ser así, ¿Cómo se caracterizaba en los casos particulares? ¿Cómo se mantenía? ¿Por qué? ¿A quién servía y cómo? ¿Cómo se vinculaba con nuestro supuesto intento de modernización como país, como sociedad? ¿Qué papel jugaba la historia del encuentro de nuestras culturas constitutivas en este asunto? —esas y otras eran preguntas que salían al paso del inicio de nuestro andar académico.

Obviamente esas preguntas estaban dirigidas a buscar una mayor compresión del modo de ser de nuestras instituciones latinoamericanas y de las formas

\footnotetext{
5 En los tiempos que corren, en los que la "imagen institucional" se convierte en una preocupación central y en los que el afán de éxito ha llegado al punto de considerar que la apariencia de éxito y éxito son lo mismo, la "esquizofrenia institucional” parece ser una fenómeno endémico en toda la cultura occidental y occidentalizada.
}

culturales que las determinaban. Pero más allá de esto, estaban dirigidas a entender el sentido de esa idea de “desarrollo" que parecía determinar los discursos oficiales que justificaban la existencia de tales instituciones (Fuenmayor, 2000b). Por el contrario, la mentalidad instrumental que dominaba por doquier simplemente se preocupaba por hacer más eficiente estas instituciones en su ciega tarea de contribuir con el crecimiento económico y la industrialización.

Resulta ahora claro que esta segunda inquietud académica también estaba, al igual que la primera, acompañada por un interés político (en el sentido original de la palabra): considerábamos que tendríamos mejores organizaciones en la medida en que estuviésemos en mayor capacidad de discutir sus sentidos actuales y deseables.

En fin, el dominio del interés y mentalidad instrumental se convirtió en una potente fuerza opositora ante nuestros dos propósitos básicos: construir una fundamentación teórica para el enfoque de sistemas y comprender el ser cultural de nuestras instituciones latinoamericanas. Aquellos dos propósitos se encontraron fácilmente en la formulación de un gran proyecto...

\subsection{El proyecto derivado de la reunión de las dos inquietudes: Sus lados “constructivo” y "combativo"}

Las dos inquietudes —la fundamentación del enfoque de sistemas y la comprensión del fenómeno de esquizofrenia institucional- se encontraron fácilmente en la formulación de un ambicioso proyecto de investigación; el cual realmente se convirtió en un proyecto de vida académica: Desarrollar una teoría de sistemas a partir de la cual fuera posible estudiar el sentido socio-cultural de las instituciones y prácticas sociales de nuestro medio.

Este proyecto marcaba el curso de lo que debía ser nuestro trabajo de estudio e investigación. Pero, así formulado, sólo representaba lo que podríamos llamar el lado "constructivo" o "positivo" del proyecto, ya que, visto por su otra cara —llamémosla “combativa” o "negativa" ${ }^{6}$ - el proyecto era, al mismo tiempo, una "batalla" contra el instrumentalismo dominante.

Permítaseme unas palabras sobre, por una parte, el carácter de este "batalla" y de sus "frentes de combate”, y por la otra, sobre la relación entre la forma combativa del proyecto y su forma "constructiva” (entre el "lado negativo" y el "lado positivo") — características propias de nuestra actividad en aquellos años.

\subsubsection{La "batalla" contra el instrumentalismo.}

La "batalla" contra el instrumentalismo se daba en diversos frentes y contra varias de sus formas - frentes y formas que no eran fácilmente separables entre sí. Voy

\footnotetext{
${ }^{6}$ Note el lector que los términos "positivo” y "negativo” tienen aquí una connotación dialéctica que para nada refleja el uso moralista y psicologista que está de moda en estos días en relación con dichos términos: Aquí "positivo" no significa "bueno" versus un "negativo" “malo”. Tampoco "positivo” significa "emprendedor”, “optimista”, versus un "negativo” "apático”, "pesimista”, “derrotista”. En nuestro contexto positivo está más vinculado con lo aparente y con el propósito final y lo negativo con lo oculto y con la lucha contra los obstáculos que se le presentan y que, al mismo tiempo, definen la forma "positiva".
} 
a mencionar a continuación dos de estas formas de instrumentalismo y apuntar algunos de los frentes asociados a cada una de ellas:

La forma más llana es aquella que rechaza todo intento de comprensión que no sea un medio para alcanzar una meta pre-definida e incuestionada. El frente más cercano de esta forma lo conseguíamos en la propia Escuela de Ingeniería de Sistemas; y, un poco más alla: en la Facultad de Ingeniería, en la Universidad de Los Andes, en la calle, en los medios de comunicación, en los libros, en la idea de "desarrollo", entre los amigos, entre los familiares. Otros frentes dentro de la misma forma aparecían en el ambiente internacional vinculado al pensamiento y práctica de sistemas, en medios de comunicación de otros países, en las relaciones internacionales, en la economía, en el consumo, en el mercado..

Otra forma del instrumentalismo lo constituía, por una parte, la omni-presencia socio-cultural de la tecnología y, por la otra, la justificación tecnológica de la ciencia. Como sabemos, esta justificación tecnológica ha venido a ocupar la razón de ser de la actividad científica, no sólo en la masa de la población, sino, también, en la mente de la mayor parte de los científicos y de los administradores de las finanzas de la actividad científica. Los frentes correspondientes a la justificación tecnológica de la actividad científica se constituían cada vez que debíamos dar cuenta de nuestra actividad académica ante los organismos burocráticos del caso. En relación con la omnipresencia general de la tecnología, el frente de batalla fue apareciendo en la medida que avanzamos por nuestra senda de pensamiento. Fuimos comprendiendo que la condición de posibilidad de la tecnología contemporánea es un modo de relacionarse con la realidad, que cada vez adquiere mayor dominio, y de acuerdo con el cual cualquier cosa que sea el caso, material o inmaterial, se constituye en un dispositivo listo para ser usado (como si fuera un instrumento tecnológico más).

Ciertamente la "batalla" tenía sus episodios de enfrentamientos dialógicos con colegas y otras personas; pero fundamentalmente se daba en nosotros mismo, en nuestras dudas, en nuestro estudio de muchos campos del saber que nos reclamaban urgentemente: particularmente, la filosofía, la sociología, la antropología, la psicología y la economía.

El lado negativo de nuestra actividad, la "batalla", desde el comienzo hacía aflorar el carácter político de toda nuestra empresa. Digo político, porque en el fondo era un debate (más interno que externo) sobre lo que es bueno y lo que es malo para las personas, para la sociedad venezolana, para las sociedades latinoamericanas, para el mundo. Además, y esto es de fundamental importancia, esa "batalla" condicionaba y era condicionada por el lado "positivo", por la forma visible del proyecto, por su lado constructivo, por el plan, por la esperanza, por el sueño. Vienen al caso unas palabras sobre este lado positivo:

\subsubsection{La onto-epistemología para el enfoque de sistemas.}

El proyecto, nuestro proyecto, era un camino que se abría ante nosotros invitando a caminarlo. No tengo aquí tiempo para contar el curso de ese camino, ni siquiera resumirlo manteniendo un hilo conductor. Sólo me limitaré a indicar uno que otro pasaje de ese apasionante viaje.

Recordemos las dos inquietudes que, juntas, empujaban aquel caminar: desplegar una ontoepistemología para el enfoque de sistemas y comprender la condición de ser de nuestras instituciones públicas. La primera inquietud nos condujo muy pronto a la idea de que la condición holística de algo es su sentido. El todo que esa silla constituye es su sentido. El todo que una institución como la universidad constituye es su sentido. Pero el sentido es, descubrimos con gran asombro, una función trascendente en relación con aquello de cuyo sentido hablamos. El sentido de la silla (o de la universidad) trasciende la silla (o la universidad). El sentido de la silla trasciende hacia algo más que no es la silla. El sentido de $x$ no se da dentro de $x$, no es inmanente a $x$, trasciende a $x$, lo trasciende hacia... ¿hacia qué? - “hacia nosotros”, era la respuesta más simple que nos venía a la mente. Pronto descubrimos otra cara de esa trascendencia: la dependencia ontológica de lo que se presenta en relación con un fondo que no se presenta, pero que lo hace posible. Esa otra cara nos enseñó que la "fuga” a la que se refería la primera cara de la trascendencia no era simplemente hacia "nosotros" como individuos, sino a nosotros como cultura, como historia encaminada hacia un por-venir.

Ingenieros de formación —estábamos ante un descubrimiento que, por decir lo menos, era vertiginoso: Si es cierto que la condición holística de algo (su ser) es su sentido, y el sentido de ese algo trasciende el algo, entonces debíamos concluir que: ¡el ser de algo trasciende ese algo! ¡Una cosa no es igual a sí misma! El ser de las cosas se fuga continuamente fuera de las cosas, pero sin abandonar las cosas.

Esta condición trascendente del ser de las cosas consistía en su dependencia ontológica de un fondo que le brinda su ser a cada cosa — como el fondo le brinda su ser a la figura de la Gestalt.

Éste era un pasaje muy significativo de aquel viaje; pero para poder conectarlo con el propósito de este cuento debo esbozar el camino donde tal pasaje ocurría:

Dada la oposición dialéctica que parecía sostenerse entre un enfoque de sistemas y el enfoque reduccionistaanalítico que parecía dominar el modo de ver científico y tecnológico, consideramos que el camino que abría la pregunta por la condición de posibilidad de la trascendencia holística debía ir al lado del camino que había sido abierto por la siguiente pregunta: ¿Por qué la tradición científica occidental ha ignorado la trascendencia holística de los fenómenos? El camino que abría esta última pregunta era el del despliegue de la onto-epistemología del reduccionismo. El otro era el del despliegue de la onto-epistemología de sistemas. Ambos se irían construyendo a partir de su tensión dialéctica. Así, por ejemplo, luego de andar y desandar el "Discurso del Método" y las "Meditaciones" de Descartes en busca de la raíz del análisis a priori y del reduccionismo, comprendimos que la ontoepistemología sistémica debía vacunarse contra ese desliz cartesiano de pretender derivar lo pensado del pensamiento (el cogito ergo sum), como si el pensamiento no incluyese necesariamente lo pensado en 
él. De allí aprendimos que la más mínima ocurrencia (el más simple hecho) lleva consigo, de manera indesligable, la situación vivencial a la que pertenece y desde la que es lo que es. Aprendimos que las cosas son actos de aparición cuya forma ontológica obedece al de la distinción (gracias a Spencer-Brown por la inspiración) —a la distinción de la figura de lo que es el caso sobre el fondo que hace posible esa figura. Aprendimos que ese fondo, indistinguible por definición, ha sido dejado allí por la experiencia del vivir; del vivir, no sólo nuestro, sino de lo que nos antecedió: de nuestra historia colectiva. Puesto en otras palabras: nos percatamos que el fondo que hace posible la forma de cualquier cosa o hecho es de carácter histórico-cultural. Descubrimos así la condición histórica-ontológica de todo acontecer. Y ese descubrimiento ya era el inicio de la segunda etapa... Pero antes de pasar a comentar esa segunda etapa, debo recapitular sobre lo logrado en la primera.

Al final de la década de los años ochenta contábamos con una onto-epistemología para el enfoque de sistemas ${ }^{7}$ (Fuenmayor, 1991b y 1991c) construida dialécticamente a partir de su distinción de una contraonto-epistemología que da cuenta del reduccionismo y de sus raíces onto-epistemológicas más profundas en el origen del pensamiento Occidental (Fuenmayor, 1991a).

Pero la segunda interrogante también había dados sus frutos:

2.3.3 La teoría sistémico-interpretativa de organizaciones y la teoría de diseño.

Con respecto a la segunda interrogante de la primera etapa, habíamos desarrollado una “Teoría sistémico-interpretativa de organizaciones" ${ }^{8}$ basada en la onto-epistemología, la cual servía de base teórica y metodológica para los estudios de organizaciones, y una teoría general del diseño sistémico interpretativo (López-Garay, 1986). Uno de los primeros estudios fundado en estas contribuciones teóricas fue un estudio sobre el "Sentido y sin-sentido del desarrollo" publicado unos años más tarde en forma de libro (Fuenmayor, 2000b). A esto se le añadieron varios estudios de organizaciones específicas ${ }^{9}$ y unas tres decenas de artículos vinculados con la problemática de la Sistemología Interpretativa publicados en revistas de circulación internacional ${ }^{10}$.

Ya a esa altura de nuestro andar, unos cuantos años después de cruzar aquel umbral antes descrito, con una onto-epistemología sistémico-interpretativa, una teoría de organizaciones y una teoría del diseño en nuestro haber,

\footnotetext{
7 Esta onto-epistemología fue resumida y presentada al público internacional en una edición especial de la revista "Systems Practice" (Vol. 4, No. 5, 1991) dedicada a la Sistemología Interpretativa. Los cuatro primeros artículos de esta revista (Fuenmayor y López-Garay, 1991; Fuenmayor, 1991a, 1991b y 1991c) presentaron la ontoepistemología de la Sistemología Interpretativa. El resto de los artículos de este número especial de Systems Practice daban muestras de estudios organizacionales específicos organizados a partir de la teoría sistémico-interpretativa de organizaciones.

${ }^{8}$ Publicada un poco más adelante en forma de libro (Fuenmayor, 2001a)

${ }^{9}$ Por ejemplo: Dávila (1995, 1999), Fuenmayor R.L y Fuenmayor A.C. (1999), Fuenmayor A.C. y Fuenmayor R.L (1999), López-Garay, H. \& Suárez, R.T. (1999), Ochoa (1998a, 1998b, 2001), Suárez, R.T. (1999a, 1999b, 2000)

${ }^{10}$ Por ejemplo: Dávila (1997), López (1986)
}

comenzábamos a descubrir que el problema que enfrentábamos era mucho más que el de la construcción de una onto-epistemología...

\section{Segunda etapa de la sistemología interpretativa: La historia ontológica y el proyecto de educación}

Tanto el desarrollo teórico como la realización de los estudios específicos de organizaciones fueron poniendo de manifiesto un gran problema que podría ser formulado del siguiente modo: La evasión de la comprensión del sentido holístico de los fenómenos no es exclusiva de la ciencia reduccionista; se trata, más bien, de la manifestación fundamental de la crisis final de toda la cultura Occidental en el presente. Tal crisis se hace especialmente aguda en las sociedades "occidentalizadas", cuyas formas culturales tradicionales fueron destruidas sin lograrse cultivar plenamente la cultura moderna europea en su seno.

En efecto, por diferentes caminos propios del campo de investigación y reflexión que veníamos labrando, fuimos llegando a la conclusión de que el reduccionismo y el análisis prematuro que caracteriza a la ciencia, ${ }^{11}$ el cual explica la desatención del sentido de los fenómenos por parte de la ciencia dualista-reduccionista, es sólo una consecuencia cultural de un problema mucho mayor que carcome el tuétano de la cultura Occidental. En medio de nuestra perplejidad, se nos fue haciendo aparente que la vida en la cultura occidental está signada por la pobreza del sentido de lo que ocurre y por la fragmentación del sentido de la vida colectiva $e$ individual. Los caminos que nos condujeron a esta tesis eran muy variados: Algunos se derivaban de los resortes más profundos de nuestra propia onto-epistemología; otros provenían de nuestros estudios de organizaciones específicas; y otros provenían de la reflexión sobre nuestra vida cotidiana —reflexión fundada en el pensamiento holístico que fuimos aprendiendo con nuestra labor académica

\subsection{La investigación histórico-ontológica del presente.}

Surgió así una nueva gran pregunta que inauguró lo que denominamos "segunda etapa de la Sistemología Interpretativa”. La pregunta inaugural de esta etapa era comparable en su significación y en las consecuencias que debería traer a la de la primera etapa (¿Cómo es posible la trascendencia del todo sobre las partes y el estudio de tal trascendencia?). La nueva pregunta interrogaba por la condición de posibilidad de esa situación epocal que habíamos descubierto: ¿Cómo llegamos a habitar un mundo en el que lo que se presenta lo hace bajo un sentido empobrecido $y$ fragmentado? ¿Cuáles son los resortes internos que sostienen esta situación epocal? ¿Cuáles son las formas y las consecuencias del sin-sentido?

El nuevo preguntar nos envolvió, nos cautivó, nos hizo estudiar, pensar, escribir... ${ }^{12}$ Comenzamos, de este

\footnotetext{
${ }^{11}$ Contra el cual habíamos ofrecido una onto-epistemología sistémica. 12 Algunos de los escritos propios de la investigación correspondiente a la segunda etapa son los siguientes: Dávila (1993, 2004, 2006a, 2006b, 2001), Fuenmayor (1994a, 1994b, 1994c, 1997a, 1997b, 2000a, 2001b, 2001c, 2001d,), Ochoa (2000, 2004a, 2004b), Suárez (1998, 2000), Villarreal (2005). La versión más completa del resultado de esta investigación puede verse en Fuenmayor (2016)
} 
modo, a asomarnos tímidamente en la comprensión de lo que, por otros caminos, ha ocupado el pensamiento de buena parte de los mejores filósofos del Siglo XX. ${ }^{13} \mathrm{El}$ inicio en tal comprensión fue haciendo más acuciante otra pregunta que, debajo de la anterior, la empujaba, la reforzaba. La pregunta práctica ya no podía hacerse esperar más: ¿Qué se puede hacer? ¿Qué podemos hacer ante el advenimiento de una época signada por la pobreza del sentido de lo que ocurre y por la fragmentación del sentido de la vida colectiva $e$ individual?

El aspecto más de fondo de una primera posible respuesta la conseguimos en nuestra propia ontoepistemología y en la obra de algunos de los filósofos estudiados, particularmente en la obra de Martin Heidegger. Puesto en nuestros términos sistemológicos y de manera muy escueta, la posible respuesta podría tomar la siguiente forma:

El problema al que nos enfrentamos es uno de pobreza y fragmentación de sentido. De acuerdo con nuestra ontología, esto significa que se trata de un problema de pobreza del "fondo" sobre el que se dibuja cada "distinción”. Es decir, se trata de un problema de pobreza de ese fondo histórico-cultural sobre el que se dibuja la forma de cualquier cosa que sea el caso. Puesto en términos mucho más simples y cotidianos: El dar cuenta del sentido de lo particular (e.g. ¿por qué estoy aquí en este momento haciendo lo que estoy haciendo?) toma, cuando efectivamente se quiere dar cuenta de la particularidad del caso, una forma narrativa (e.g. el cuento de cómo llegué aquí para hacer lo que estoy haciendo). Y esto es así porque ese dar cuenta del sentido no es otra cosa que dar cuenta de ciertas "regiones pertinentes" del fondo histórico que le brinda su sentido al presente. Esa es la razón, sea dicho de paso, por la cual, para dar cuenta de nuestro Proyecto de Educación, hemos estado narrando el devenir de la Sistemología Interpretativa, del cual surge dicho proyecto.

Es así como la idea seminal de un proyecto de educación básica destinado a enriquecer el sentido de la vida y del acontecer llegó a estar sobre el tapete: Enseñar de manera tal que lo que se enseñara tuviera sentido, y que, en la medida de lo posible, se generara en el aprendiz el hábito de buscar el sentido de lo que le acontece en la vida. Tal hábito de vivir una vida plena de sentido podría conducir a que la pregunta sobre cómo llegamos al sin-sentido de presente encuentre un sentido histórico-ontológico que rompa el círculo vicioso del sin-sentido.

\subsection{El Proyecto de Educación de la Sistemología Interpretativa.}

Pero, ¿cómo enseñar de manera que lo que se enseñe tenga sentido, y que, en la medida de lo posible,

\footnotetext{
${ }^{13}$ En efecto, en este siglo, la obra de pensadores tan diversos como Martin Heidegger (1971, 1977a, 1977b, 1977c, 1977d, 1982, 1985, 1989, 1993a, 1993b, 1993c), Ludwig Wittgenstein (1978), T. Adorno (1966), Michel Foucault (1968, 1984), J.F Lyotard (1984), Alasdair MacIntyre (1981, 1988, 1990), Charles Taylor (1989), Jürgen Habermas (1992), Richard Rorty (1980), Gilles Deleuze (1983), J. Baudrillard (1988) constituye un rico aporte para la gran tarea del presente - tarea que, sea dicho de paso, ha quedado reservada para una reducida elite intelectual con poca resonancia fuera de ella.
}

se genere en el aprendiz el hábito de buscar el sentido de lo que le acontece en la vida? A su vez, esta pregunta, la cual en lo que sigue denominaremos pregunta práctica fundamental, conducía a otra:

Ya hemos explicado que en nuestra segunda etapa encontramos que todo el ambiente cultural de las culturas occidentales del presente, y muy especialmente de las que llamamos occidentalizadas, está dominado por la pobreza y fragmentación del sentido de lo que ocurre. La educación formal, familiar, laboral, y la que tiene lugar en cualquier forma social no puede escapar a tan profunda problemática. Con la pregunta práctica fundamental en mente, preguntamos: ¿Qué hay en el modo usual de enseñar propio de la educación formal que se presta para perpetuar el sin-sentido? - A ésta, llamémosla: Primera pregunta práctica derivada

Estas preguntas y muchas otras guiaron el Proyecto de Educación de la Sistemología Interpretativa. Primero ocurrió una etapa de diseño de material didáctico que describiremos en la siguiente sub-sección. Luego pasamos a una etapa de investigación-acción mediante la creación y funcionamiento de una escuela piloto que le dimos el nombre de "Paideia". Es conveniente aclarar que la respuesta más acabada que hoy tenemos ante la anterior pregunta práctica fundamental y su primera pregunta práctica derivada fueron el producto, por una parte, del estudio de una serie de textos, particularmente aquellos referentes a desarrollos en la filosofía del lenguaje del último siglo. Pero también fueron producto de la reflexión sobre la primera etapa de diseño y la reflexión sobre el quehacer en la escuela Paideia. Así se fue constituyendo una triada recursiva constituida por: 1) el estudio de textos, 2) las actividades de diseño de material didáctico y la actividad escolar, y, 3) la reflexión sobre el diseño, sobre la actividad diaria escolar y sobre la vinculación entre esa reflexión y lo que estudiábamos en aquellos textos. El estudio alimentaba la reflexión sobre la acción, y la reflexión sobre la acción nos ayudaba a darle sentido al estudio de los textos. A su vez, la reflexión guiaba la acción y la acción permitía la reflexión sobre la misma y sobre la vinculación con lo estudiado en los textos.

3.2.1 Los primeros pasos en el Proyecto de Educación de la Sistemología Interpretativa: la etapa de diseño de material didáctico.

Habíamos dicho que, de acuerdo con lo indagado en la segunda etapa de la Sistemología Interpretativa, las sociedades occidentales y occidentalizadas sufren una severa, sistémica y profunda enfermedad: la pobreza y fragmentación del sentido del acontecer. ¿Cómo enseñar de manera que lo que se pretende enseñar tenga sentido, es decir, que realmente se aprenda? Pero, más allá y más difícil: ¿Cómo enseñar de manera que el aprendiz cree el hábito de buscar el sentido del acontecer?

La idea básica con la que comenzamos el proyecto fue la de diseñar textos contentivos de narrativas ${ }^{14}$ en los que se insertaran, a modo de "paréntesis" o "ramificaciones", habilidades-conocimientos vinculados con las temáticas que aparecen en los programas

\footnotetext{
${ }^{14}$ La cuales cubrían un rango que iba desde cuentos muy sencillos, pero bien escritos, para los más pequeños, hasta novelas de buena literatura para los mayores.
} 
oficiales. Así, a propósito de diferentes ocurrencias que aparecieran en la narrativa, se insertarían prácticas que permitieran iniciar el entrenamiento del niño en los juegos lingüísticos ${ }^{15}$ de las matemáticas, la biología, la física, el arte, la historia, etc. Es decir, se buscaba que mientras se desarrollaba el juego lingüístico narrativo, se insertara en su interior el desarrollo de esos otros juegos lingüísticos. De esa manera, se entrenaría el niño en el hábito de buscar un sentido narrativo del saber, de manera que permitiera que tales habilidadesconocimientos no sólo tuviesen sentido por sí mismos, sino pudiesen insertarse en el hilo conductor de la vida.

El diseño inicial contempló varias tareas:

1) Diseñar una especie de "guion histórico" general que proporcione una primera forma básica de una narrativa matriz (la que da cuenta del presente). Esto no sería otra cosa que una posible narración históricoontológica sobre cómo llegó a constituirse nuestro presente en el modo como se nos presenta. La madeja narrativa aportada por la filosofía contemporánea encontraría su campo de debate en esa narración - tanto para encajar en él como para cuestionarlo e irlo modificando.

2) Mostrar cómo, a la luz de ese guion histórico, se puede ver que las grandes revoluciones educativas de la historia Occidental han obedecido a la necesidad de lidiar con cambios epocales —asociados estos, a su vez, a cambios en la narrativa matriz. El resultado de la investigación-diseño concerniente a estas dos primeras tareas nos serviría de trasfondo para alimentar las narrativas y nuestro comportamiento docente general, y para usarlo de material narrativo en etapas superiores de la educación.

3) El diseño propiamente dicho (alimentado continuamente por las otras dos actividades antes descritas) de un sistema educativo para la escuela de la Venezuela del presente. La idea que subyacía esta primera etapa del diseño era la siguiente: La columna vertebral de todo el proceso de enseñanza-aprendizaje sería la lectura de cuentos - al principio, simples cuentos infantiles, luego y gradualmente, cuentos que comienzan a vincularse más directamente con la narrativa mencionada en (1). En esta lectura de cuentos

\footnotetext{
${ }^{15}$ Estoy usando esta frase en el sentido que entiendo es usada en el libro "Investigaciones Filosóficas" de Ludwig Wittgenstein (1978). Uno de los ejemplos que Wittgenstein usa para caracterizar lo que llama "juego lingüístico" son esos juegos infantiles en los que los niños cantan una canción mientras realizan, en conjunto, una serie de actividades (“acciones dentro de las cuales se teje un lenguaje” p. 5) relacionadas con la letra de la canción (e.g. "A la víbora de la mar”) En general pienso que se trata de un modo de hablar "entretejido" con un modo de actuar (o, un modo de actuar entretejido con un modo de hablar), diferente de otros modos de "hablar - actuar", en el que el modo de hablar le da sentido al modo de actuar (además, lo comanda, comenta, reporta, proyecta); y en el que el modo de actuar realiza, le da sentido, concreción y ubicación al modo de hablar; de manera que no es posible ni pensar ni vivir el modo de actuar sin el modo de hablar, ni el modo de hablar sin el modo de actuar. La unidad indisoluble que ellos constituyen se llama “juego lingüístico”. En un "juego lingüístico", las palabras son comprensibles en términos de contexto brindado por todo el "juego lingüístico". Y este "ser comprensible" no significa que ellas "signifiquen” algo en el juego lingüístico (como una palabra que buscamos en un diccionario “significa” su definición), sino que tienen un (o unos) "uso(s)” en el "juego lingüístico".
}

se distinguirían dos actividades fundamentales que debían estar presentes a lo largo de todo el proceso.

3.1 Cultivo de los cuentos: Tenía por objetivo lograr destreza en el lenguaje y en las habilidades intelectuales básicas. Esta actividad estaría constituida por subactividades tales como las siguientes: luego de una primera lectura, cada cuento se contaría y comentaría reiteradamente, en lenguaje oral y escrito por parte de maestros y alumnos; se resumirían los cuentos; se discutirían sus interpretaciones; se obtendrían sus moralejas; se construirían nuevos cuentos a partir de los originalmente leídos; se emprenderían otras actividades en torno a los cuentos tales como dibujos, bailes, cantos, excursiones, etc.

3.2 Derivación de contenidos temáticos a propósito de los cuentos: A lo largo de la lectura y del cultivo de los cuentos, se introducirían esos temas que, en el actual sistema educativo, se encuentran ubicados dentro de materias tales como matemáticas, física, historia, etc. Sin embargo, desde el punto de mira de nuestra práctica escolar, serían presentados como partes de los cuentos que servirían el propósito inmediato de enriquecer la comprensión de los mismos. De este modo, bajo la perspectiva narrativa y aprovechando su poder integrador, se irían introduciendo los temas que hoy constituyen los programas de estudio. Esto significa que, en un momento dado, dentro del contexto de un cuento específico, la actividad escolar se abocaría, por ejemplo, a comprender qué son los números fraccionarios y cómo operar con ellos.

El diseño del proceso educativo en torno a cuentos se propuso crear textos guías que contuvieran de manera muy específica y detallada todo lo que se iría haciendo en el salón de clases. La razón de esta especificidad descansó en que partimos del hecho de la muy mala preparación de nuestros maestros. El texto guía le serviría al maestro para aprender mientras enseña. ${ }^{16}$

Esa primera etapa de diseño duró aproximadamente cuatro años, al cabo de los cuales disponíamos de suficiente material didáctico (punto 3 del diseño), y suficiente adelanto en la investigación históricoontológica referida en los puntos 1 y 2 del diseño, para iniciar una escuela piloto en la cual poner en práctica las ideas así gestadas. ${ }^{17} \mathrm{La}$ actividad escolar nos aportaría importantes lecciones que enriquecerían considerablemente el diseño inicial. Veamos:

\subsubsection{Paideia: Educación con sentido.}

Ése fue el título de un documental sobre nuestra escuela piloto (https://www.youtube.com/watch?v=_QWTdnz6LNg), a la cual bautizamos con el nombre de "Paideia".

En efecto, después de unos años dedicados a la gestación de las ideas básicas de ese modo de enseñanza, iniciamos las labores educativas en una escuela piloto que denominamos Paideia. Allí, durante un período de diez años, pusimos en práctica esas ideas iniciales y

\footnotetext{
${ }^{16}$ Ejemplos de otros escritos resultantes de la etapa inicial del Proyecto de Educación de la Sistemología Interpretativa son: Suárez (2003, 2004), Villarreal (2006).

${ }_{17}$ Estos resultados pueden verse principalmente en Crespo (2014), Suárez (2005), y Villarreal (2007). Aparte de estas tres tesis doctorales se concluyeron otras tesis de maestría y de pregrado que contienen contribuciones para el diseño del material didáctico.
} 
otras que fueron surgiendo a partir de la experiencia ganada mediante la actividad escolar dentro de un ambiente de investigación-acción; ${ }^{18}$ las cuales no simplemente se añadieron a las iniciales, sino que también las transformaron.

Una de esas nuevas ideas fue la realización de una serie de juegos en los que los niños participaban y en los que, en la mayor parte de los casos, desempeñaban una actividad física reunida con actividades que acostumbramos a llamar mentales, las cuales involucraban una serie de habilidades-conocimientos que se querían enseñar. ${ }^{19}$ En la práctica educativa usual se separan artificialmente los conocimientos de los juegos lingüísticos en los que pueden tener lugar. Por el contrario, en estos juegos practicados en Paideia, ambos tipos de destrezas, físicas y mentales, reunidas en una sola actividad, confluían en el lenguaje básico del niño para lograr las metas de los juegos. ${ }^{20}$ De este modo se iban construyendo juegos lingüísticos más especializados vinculados con la lógica, la matemática y otras disciplinas, sobre la base del lenguaje básico del niño, el cual, a su vez, se iba enriqueciendo. Todo ello se daba dentro de un ambiente lúdico, agradable para el aprendiz, y con gran poder para enseñar el sentido de lo que se aprende.

Aparte de estos juegos había múltiples actividades, muchas vinculadas con las artes, otras dedicadas a la solución y discusión de problemáticas de diferentes tipos (matemático-físicas, químicas, morales, etc.), lectura y cultivo de cuentos. Además, se realizaban excursiones a sitios naturales, asistencia a conciertos, visitas a museos, hospitales, lugares urbanos particulares...

No sé si estos niños que permanecieron en Paideia durante un cierto número de años —algunos de los cuales, ya iniciaron sus estudios universitarios- habrán generado las condiciones para preguntarse cómo se gestó el modo histórico-ontológico dentro del cual actualmente nos relacionamos con los otros, lo otro y con nosotros mismos. Pero sí sé que se logró impartir un modo de enseñar que despertó la curiosidad del niño por el sentido vital de lo que allí se impartía, en el que el aprender fue un placer para el niño y el enseñar un placer para el maestro, y, muy importante para la problemática moral de esta época a-moral que vivimos, se logró crear un ambiente socio-cultural escolar signado por la fraternidad y por el deseo de aprender. Con respecto al aspecto más tangible de naturaleza típicamente cognoscitivo, menciono, por ejemplo, un hecho objetivo en relación con el aprendizaje de los juegos lingüísticos propios del programa escolar: Durante varios años, Paideia fue la escuela venezolana que obtuvo el mayor número de medallas por

\footnotetext{
${ }^{18}$ Véase al respecto: Fuenmayor (2001e y 2016), Dávila (2016) y el documental sobre la escuela Paideia antes aludido: https://www.youtube.com/watch?v=_QWTdnz6LNg.

${ }^{19}$ Como estos juegos implicaban exigentes actividades físicas (carrera, salto, etc.), los realizábamos en un amplio patio central y los llamábamos "juegos del patio".

${ }^{20}$ Recuérdese que el "lenguaje básico" es el sistema de juegos lingüísticos básicos de una cierta forma socio-cultural, por tanto tiene la forma de habla-acción propia de los juegos lingüísticos.
}

estudiante ${ }^{21}$ en las olimpíadas nacionales de matemáticas en Venezuela.

Los niños que permanecieron varios años en Paideia y después fueron a la Universidad, no sólo han tenido éxito en sus estudios, algunas veces un éxito extraordinario, sino que han mantenido una honesta actitud de cuidado de los otros y de lo otro.

Pero, detengamos la narración para volver a la pregunta práctica fundamental que guió este proyecto de manera de dar cuenta, muy brevemente, de la respuesta conseguida a lo largo de todo el proceso descrito:

\subsubsection{El fundamento del modo de enseñar en Paideia.}

Recordemos esa pregunta práctica fundamental que trajo consigo la creación de la escuela piloto Paideia: ¿Cómo enseñar de manera tal que lo que se enseñe tenga sentido, y que, en la medida de lo posible, se genere en el aprendiz el hábito de buscar el sentido de lo que le acontece en la vida? $\mathrm{Y}$ su derivación inmediata: ¿Qué hay en el modo usual de enseñar propio de la educación formal que se presta para perpetuar el sin-sentido? Intentemos una respuesta a esta primera pregunta práctica derivada:

Detrás de la mayor parte del pensamiento de la modernidad y de sus precursores hay una cierta forma de interpretar la condición "racional” del ser humano que incidió directamente en el modo de concebir la educación que ha dominado desde entonces. Esta interpretación de la condición racional del ser humano ha jugado un papel fundamental en el modo de entender la relación entre el pensamiento reflexivo o uso del intelecto y la acción humana (propio, por ejemplo, de lo que Kant consideraba como la razón práctica), la cual sigue dominando en la actualidad en buena parte de las prácticas educativas. Se trata de la relación que llamaremos de “aplicación”: aplicación del conocimiento obtenido intelectualmente en situaciones específicas. En consecuencia, llamaremos intelectualismo(s) a la(s) corriente(s) de pensamiento que se deriva(n) de la hegemonía de esta noción de aplicación.

En efecto, la creencia que sustenta esta noción de “aplicación” se puede expresar en dos puntos o pasos:

1) El ser humano tiene la capacidad de apropiarse del conocimiento mediante la escucha de discursos orales explicativos o la lectura de textos donde se expliquen tales conocimientos. Basta que atienda $\mathrm{y}$ estudie lo oído o leído para que pueda actuar de acuerdo con las indicaciones derivadas de tal conocimiento; es decir: pueda "aplicar” el conocimiento en una situación vital específica. Esta aplicación será siempre una consecuencia mediata (no inmediata) del acto intelectual: es decir, primero pensará y después aplicará el resultado de lo pensado a la acción.

2) Es posible y deseable que todo el actuar humano, por lo menos el que se da en el ámbito de influencia de las disciplinas enseñadas en la educación formal, sea el producto de ese modo aplicativo de entender la relación entre ese tipo de conocimiento intelectual y la acción humana.

\footnotetext{
${ }^{21}$ Me refiero al cociente entre el número de medallas obtenidas en cada escuela dividido entre el número de alumnos de esa escuela.
} 
Como se puede ver, no sólo se trata de una creencia que afecta la educación; es una creencia sobre la naturaleza del ser humano, la cual llamamos "intelectualismo" porque le da un papel protagónico al intelecto en la conducción usual de la acción humana.

La consecuencia inmediata de del intelectualismo en los procesos educativos es el modo de educación dominante que subsiste hasta nuestros días, según el cual se imparten conocimientos mediante explicaciones conceptuales, luego de las cuales el estudiante deberá estar capacitado para aplicar los conocimientos obtenidos de esa manera.

Esta creencia y consecuencia pedagógica ignora algo que, gracias a importantes desarrollos realizados en diferentes disciplinas (tales como la filosofía, la antropología, la lingüística, la pedagogía y, más recientemente, la neurofisiología), sabemos hoy: La mayor parte de nuestras acciones no son, ni pueden ser, consecuencia lo que hemos llamado "aplicación”. Son consecuencia de procesos de entrenamiento que generan en nosotros modos de actuar sobre los que generalmente no tenemos un comando intelectual. Claro está, esto no quiere decir que, en ciertas ocasiones especiales, no podamos realizar "aplicaciones".

En efecto, nuestro apreciar-actuar es como el del jugador de futbol, el cual aprecia la situación en la que recibe un balón y, en el mismo acto de apreciar-actuar, lo patea con una cierta fuerza y una cierta dirección, de manera que logra un espectacular gol, el cual hubiese sido imposible para un principiante en ese deporte. El experto jugador no calculó antes ni la fuerza ni la dirección que le imprimiría al balón para luego "aplicar" el resultado de su cálculo —como sí hace, por ejemplo, un ingeniero civil cuando calcula las condiciones de una cierta estructura para luego "aplicarlas". No, el experto jugador de futbol, o de cualquier otro deporte o juego, simplemente pone en práctica su destreza ya adquirida gracias a un proceso de entrenamiento que lo transformó -y lo hace siguiendo unas reglas de las cuales no está consciente en el momento de seguirlas. Como bien lo mostró Wittgenstein (1978), eso es lo que todos hacemos cuando hablamos u oímos lo que nos dicen. En general, tal como se ha mostrado de sobra en diferentes campos del saber, ${ }^{22}$ ése es nuestro modo de apreciaractuar, o como diría Varela (1997), de enactuar en la situación vital. Pero, ésa además es la manifestación cotidiana y más frecuente de la racionalidad humana, la cual no es meramente individual, es cultural. Es decir, las racionalidades ${ }^{23}$ están impresas en la cultura y sus juegos lingüísticos. Los individuos ordinariamente actuamos de acuerdo con esas racionalidades culturales, sin que medie un acto intelectual de parte de cada uno. Claro está, lo que digo no implica que no exista el acto intelectual y la aplicación consecuente, pero este acto generalmente es extraordinario en la mayoría de los individuos y se da en unas culturas más que en otras, pero siempre de manera extraordinaria.

\footnotetext{
22 Varela, 1997 y 2011.

${ }^{23}$ Note el lector que digo "racionalidades" en plural, porque hay diferentes racionalidades en diferentes formas culturales y diferentes épocas de la misma cultura.
}

La enacción así entendida depende de nuestro entrenamiento cultural; y nuestro entrenamiento cultural depende de la cultura epocal en la que nos corresponde vivir. En otras palabras: Los modos de apreciar-actuar en cualquier situación vital se dan sobre la base de lo que en la segunda etapa de la Sistemología Interpretativa llamamos sido-siendo: el fondo histórico-cultural sobre el cual ocurre la distinción de lo que es el caso.

En general, nuestra conclusión ha sido que la enseñanza escolar debe consistir en un entrenamiento similar al que tiene lugar cuando se enseña una nueva lengua en los mejores centros destinados a ese fin, o cuando se enseña bien una disciplina deportiva, o cuando se enseña bien un oficio en una buena práctica de ese oficio. Es decir, hemos llegado al convencimiento de que la auténtica enseñanza es un proceso de entrenamiento mediante el cual se guía el aprendiz para que desarrolle las habilidades-conocimientos (son inseparables) requeridas en un cierto campo disciplinario. Se le enseña a apreciar-actuar en ese campo. ${ }^{24}$ Pero esta enseñanza tiene que partir del sidosiendo del aprendiz en cada etapa del aprendizaje. Por ejemplo, no se le puede exigir a una persona que está apenas aprendiendo a nadar que nade 2.000 metros, sin parar, en un tiempo de media hora. Su condición físicomental no se lo permitiría y lo único que lograríamos es generar en ella o en él molestia, desánimo y miedo. Asimismo, carece de sentido comenzar a enseñarle fracciones a un niño sin que haya desarrollado la intuición básica de lo que es una fracción mediante el manipular-hablar de las partes que surgen cuando se separa una unidad en partes. Es decir, es necesario sembrar en el sido-siendo del aprendiz las intuiciones básicas que le permitirán más adelante la apreciaciónacción requerida en el campo correspondiente (e.g. el de las fracciones en aritmética).

Dicho en términos lingüísticos (y siguiendo la concepción de lenguaje generada en las "Investigaciones Filosóficas” de L. Wittgenstein (1978)): en la educación escolar, ${ }^{25}$ es necesario enseñar los nuevos juegos lingüísticos sobre la base del lenguaje básico del aprendiz, de manera que lo que se enseña tenga sentido; que no se produzca la alienación y el sin-sentido resultante de enseñar "en el aire", sin partir del lenguaje básico del aprendiz. Pero, además, como decíamos antes, los nuevos juegos lingüísticos —sean estos los de las matemáticas, los de la física, los de la filosofía, los de la literatura, o cualquier otro- deben ser enseñados "jugando", es decir enactuando. Estas dos ideas enseñar sobre la base del lenguaje básico y enseñar jugando los nuevos juegos lingüísticos - constituyen lo que John Dewey (1966), ese gran pionero de una educación con sentido, llamaba (y abogaba por) una enseñanza que partiera de la experiencia del aprendiz.

Lo anterior implica el diseño de modos de interacción social con propósito didáctico mediante los cuales se pueda lograr la maestría en la habilidadconocimiento que se pretende enseñar; y en los que,

\footnotetext{
${ }^{24}$ Recuérdese los ejemplos de entrenamiento de deportistas y artesanos con los cuales ilustramos la noción de apreciación-acción.

${ }^{25}$ Cuando digo "escolar" me refiero a toda la educación impartida por instituciones dedicadas a ese fin: desde la educación primaria hasta la universitaria.
} 
además, exista la interacción con todo aquello (otros seres humanos, instrumentos, objetos de estudio, ambientes físicos, etc.) que se relaciona con la disciplina del caso. Pero toda esa interacción debe ser armónica, signada por el cuidado de los otros y de lo otro. Tales modos de interacción social que acostumbro a llamar juegos $^{26}$ deberían ser la base de la enseñanza: Se enseñaría jugando estos juegos. Las explicaciones, las cuales actualmente ocupan la mayor parte de la actividad docente, deben ser (como lo han sido en Paideia) apenas una parte, y no la más importante, de la introducción y conducción de esos juegos.

Para terminar, vale la pena anotar que este asunto de la diferencia entre el modo de apreciar-actuar que estamos describiendo y el intelectualismo tiene una incidencia directa en la discusión sobre nuestro modo de relacionarnos con situaciones que nos ofrecen tintes morales. En particular, el intelectualismo supone que la posibilidad de una acción moral con fundamento racional sólo es posible mediante un acto de aplicación: primero, pensar qué hacer, $\mathrm{y}$, después, proceder en consecuencia. Bajo nuestra noción de apreciar-actuar, y tal como ya lo planteaba Aristóteles, la posible acción moral cotidiana normalmente es producto de procesos de entrenamiento socio-cultural que tienen lugar sin que necesariamente haya una voluntad propositiva detrás de ellos. Sin embargo, también pueden ser dirigidos mediante adecuados entrenamientos educativos diseñados con un propósito específico. En cualquier caso, de manera espontánea o como producto de un proceso educativo propositivo, tales entrenamientos se "sedimentan” en el sido-siendo individual y están allí dispuestos para la acción.

\section{Conclusión.}

He dado cuenta, de manera muy resumida, del devenir de la Sistemología Interpretativa y de su quehacer actual en torno a su proyecto de educación.

El Proyecto de Educación de la Sistemología Interpretativa obtiene su sentido particular a partir del devenir de la Sistemología Interpretativa. Es posible, claro está, brindar una versión propositiva y un reporte de resultados de un tal proyecto, pero dar cuenta del sentido que le es propio, como dar cuenta de todo sentido particular de algo, requiere una narración de lo que históricamente lo hizo posible.

Es por ello que la mitad de este artículo se ha dedicado a presentar una versión resumida del devenir de la Sistemología Interpretativa, una experiencia universitaria que se inició hace unos 40 años y que ha incluido la enseñanza, la investigación y la investigación-acción. Dentro del movimiento de sistemas ha sido la única que ha gestado una fundamentación onto-epistemológica, sobre cuya base se ha erigido una teoría de organizaciones, un método para estudiar las mismas, una teoría del diseño, una experiencia de investigación en numerosas organizaciones fundada en ese método, el cual surgió de

${ }^{26}$ Dada su similitud con los que usualmente llamamos 'juegos' y teniendo en mente el concepto de juego lingüístico tomado de Wittgenstein (1978) y resumido en una nota anterior, del cual, en parte, he derivado un modo de enseñanza-aprendizaje que permita la recuperación del sentido del acontecer. esas teorías, las cuales, a su vez, estaban fundadas sobre aquella onto-epistemología.

En el recorrido narrativo por el fluir de la Sistemología Interpretativa se distinguieron dos grandes etapas:

Una primera etapa movida por la confluencia de dos problemáticas: la de la construcción de una ontoepistemología para el enfoque de sistemas que diera cuenta de la trascendencia holística de los fenómenos y la del desarrollo de una teoría de organizaciones y una teoría del diseño que permitieran comprender el llamado “fenómeno de esquizofrenia institucional” en las organizaciones públicas latinoamericanas y actuar en consecuencia.

La segunda etapa, devenida de los resultados obtenidos en la primera, se inició a partir del descubrimiento de que la gran dificultad con la que se enfrenta un enfoque de sistemas, mucho más allá del carácter reduccionista-analítico de la ciencia moderna, es el gradual empobrecimiento y fragmentación del sentido de la vida y de lo que en ella ocurre dentro de las culturas occidentales y "occidentalizadas" (como la nuestra). Esta segunda etapa condujo a una investigación histórico-ontológica sobre el modo como llegamos a ser lo que hoy somos y nuestro mundo lo que hoy es. A su vez, la pregunta práctica que subyacía el estudio y que, al mismo tiempo afloraba de éste, condujo al diseño de un proyecto de educación básica que permitiera enseñar con sentido holístico y generar el hábito de buscar el sentido de lo que acontece en la vida.

De ese modo, la narrativa nos llevó a una breve descripción del Proyecto de Educación al que condujo el devenir inquisitivo de la Sistemología Interpretativa. Allí describimos su etapa inicial de diseño de material didáctico y dimos cuenta de su escuela piloto. Explicamos que la enseñanza en esta Escuela estuvo fundada en una concepción no intelectualista de la relación entre la racionalidad y la acción humana. En efecto, nuestro estudio de diferentes disciplinas, particularmente la filosofía del lenguaje, y las lecciones obtenidas del propio proyecto de investigación-acción nos mostraron que la mayor parte de la acción humana cotidiana no obedece a la dominante concepción de aplicación que caracteriza la relación entre la racionalidad humana y la acción derivada de ésta. Fuimos descubriendo que la racionalidad que normalmente rige la acción humana individual y grupal es la racionalidad estructurada en los lenguajes humanos, y, en general, sus culturas. Que usualmente apreciamos-actuamos bajo las reglas implícitas incluidas en esas estructuras, las cuales han sido sembradas en nosotros mediante procesos de entrenamiento que se encuentran activos en cada forma socio-cultural; y que lo hacemos como hace sus jugadas el deportista entrenado para tal fin. Explicamos que los procesos educativos formales pueden aprender esta lección y proceder en consecuencia. Eso hicimos en Paideia: Mediante variados ejercicios de entrenamiento fuimos enriqueciendo el lenguaje básico de los niños y jóvenes con los juegos lingüísticos provenientes de diferentes disciplinas (literatura, matemática, ciencias naturales, arte, etc.). Tal actividad siempre se realizaba tomando en cuenta el estado del lenguaje básico de los aprendices de 
manera de sembrar en él las intuiciones básicas que permitirían erigir sobre ellas las nociones y conceptos de mayor especialización. Finalmente, se reporta el resultado general de esta experiencia educativa.

\section{REFERENCES}

[1] T. Adorno, "Negative Dialectics", Routledge, London, 1966.

[2] J. Baudrillard, "Selected Writings", Polity Press. UK, 1988.

[3] M. Crespo, "Primeros trazos de una historia-ontológica de la educación en América Latina, en la búsqueda de un sistema educativo para el presente que aspire el enriquecimiento del sentido", [Tesis doctoral], Mérida, Venezuela: Universidad de Los Andes, 2014.

[4] J. Dávila, "Foucault's Interpretive Analytics of Power", Systems Practice, 6 (4), pp. 383-405, 1993.

[5] J. Dávila, "Enmascaramiento del incumplimiento de los derechos humanos: los programas contra la pobreza en Venezuela”, Espacio Abierto, Vol IV, № 1, pp. 19-51, 1995.

[6] J. Dávila, "Un panorama actual de la Teoría de Organizaciones”, Revista Venezolana de Gerencia, No. 4, pp. 133-141, 1997.

[7] J. Dávila, "Birth and Demise of a Social Protection Organization in Venezuela", (coautoría con A. Ochoa), Systemic Practice and Action Research, Vol. 12, № 1, pp. 15-34, 1999.

[8] J. Dávila, "Ethique de la parole et jeu de la vérité", in "Foucault et la philosophie antique", F. Gros (Ed.), Ediciones Kimé, París, 2003, pp. 195-208. Versión en español: Ética de la palabra y juego de la verdad , en Foucault y la filosofía antigua, Ediciones Nueva Visión, Buenos Aires, pp. 163-174, 2004.

[9] J. Dávila, "La ética de la esperanza como esperanza de la ética”, Revista Diálogo Filosófico, España, en edición. 2006a.

[10] J. Dávila, "Actualité de la pensée de Michel Foucault pour les latinoaméricains", en Existe-t-il une philosophie latinoaméricaine?, Editions Unesco, París, pp. 39-70, 2006b.

[11] J. Dávila, “¿Ultrahumanismos?”, Revista Signos Filosóficos, $N^{\circ}$ 6, México, julio - diciembre 2001, 2001.

[12] J. Dewey, "Democracy and Education”, New York: The Free Press, 1966.

[13] J. Dávila, “Enseñar a vivir con sentido (Paideia). En Revista de Estudios Interculturales desde Latinoamérica y el Caribe”, Año: 10, No. 19, pp. 82-96, 2016.

[14] G. Deleuze, F. Guattari, "Anti-Oedipus. Capitalism and Schizophrenia", The Athlone Press. London, 1983.

[15] M. Foucault, "Las palabras y las Cosas: Una arqueología de las Ciencias Humanas”, Siglo Veintiuno Editores, México, 1968.

[16] M. Foucault, "The history of sexuality", Penguin Books. London, 1984.

[17] R. Fuenmayor and H. López-Garay, "The scene for interpretive systemology”, Syst. Pract. 4, 401-418, 1991.

[18] R. Fuenmayor, "The Roots of Reductionism: A CounterOntoepistemology for a Systems Approach”, Syst. Pract 4, 419447, 1991a.

[19] R. Fuenmayor, "The Self-Referential Structure of an EverydayLiving Situation: A Phenomenological Ontology for Interpretive Systemology", Syst. Pract 4, 449-472, $1991 \mathrm{~b}$.

[20] R. Fuenmayor, "Truth and Openness: An Epistemology for Interpretive Systemology", Syst. Pract 4, 473-490, 1991c.

[21] R. Fuenmayor, "Modernidad y Autenticidad: El no ser siendo de lo moderno", Dirección General de Cultura de la Universidad de Los Andes. Mérida, Venezuela, 1994a.

[22] R. Fuenmayor, "'Systems Science: Addressing Global Issues'-The Death Rattle of a Dying Era?", Systemist, 16, 110-157, 1994b.

[23] R. Fuenmayor, "El olvido del sentido holístico en la era postmoderna", Sistemas. No. 1, 1994c

[24] R. Fuenmayor, "Recovering Systems Thinking from Systems Thinking”, Systemist, 19 (2), 62-66), 1997a

[25] R. Fuenmayor, "The Historical Meaning of Present Systems Thinking”, Systems Research,. 14, No. 4, 235-248, $1997 \mathrm{~b}$.
[26] R. Fuenmayor, "El cultivo de la verdad", Ibagué - Colombia: Ediciones Unibagué, 2016.

[27] R. Fuenmayor, and A. Fuenmayor, "Researching-ActingReflecting On Public Health Services in Venezuela. I. A conceptual Framework", Systemic Practice and Action Research, 1999. 12, No. 1, 35-53, 1999.

[28] A. Fuenmayor, and R. Fuenmayor, "Researching-ActingReflecting On Public Health Services in Venezuela. II. Community Action and Critique”, Systemic Practice and Action Research, 12, No. 1, 55-75, 1999.

[29] R. Fuenmayor, “A Brief Crack of Light?”, Systemic Practice and Action Research, 13, No. 6, 757-772, 2000a.

[30] R. Fuenmayor, "Sentido y Sinsentido del Desarrollo", Consejo de Publicaciones de la Universidad de Los Andes, Mérida Venezuela, 2000b.

[31] R. Fuenmayor, "Interpretando Organizaciones. Una Teoría Sistémico-Interpretativa de Organizaciones”, Consejo de Publicaciones de la Universidad de Los Andes, Mérida Venezuela, 2001a.

[32] R. Fuenmayor, "The Oblivion of Churchman's Plea for a Systems Approach to World Problems. I. The Inseparability of Systems Thinking and World Issues in the Modern Epoch", Systemic Practice and Action Research, 14, No 1, 11-28, 2001b.

[33] R. Fuenmayor, “The Oblivion of Churchman's Plea for a Systems Approach to World Problems. II. The Rise of the Modern Constellation", Systemic Practice and Action Research, 14, No 1, 29-45, 2001c.

[34] R. Fuenmayor, "The Oblivion of Churchman's Plea for a Systems Approach to World Problems. III. The Fall of the Modern constellation", Systemic Practice and Action Research, 14, No 1, 47-60, 2001d.

[35] R. Fuenmayor, "Educación y la reconstitución de un lenguaje madre", Logoi, No. 4, 2001e.

[36] J. Habermas, "Postmetaphysical Thinking”, Polity Press, UK, 1992.

[37] M. Heidegger, "The Thing”, in "Poetry, Language, Thought.", Harper \& Row, Publishers, New York, pp. 165-185, 1971.

[38] M. Heidegger, "Science and Reflection", in "The Question Concerning Technology and Other Essays", Harper Torchbooks, pp. 155-182, 1977a.

[39] M. Heidegger, "The Question Concerning Technology", in "The Question Concerning Technology and Other Essays", Harper Torchbooks, pp. 3-35, 1977b.

[40] M. Heidegger, "The Turning”, in "The Question Concerning Technology and Other Essays”, Harper Torchbooks, pp. 36-49, 1977c.

[41] M. Heidegger, "Time and Being", in "On Time and Being", Harper \& Row, Publishers, New York, pp. 1-54, 1977d.

[42] M. Heidegger, "The Nature of Language", In "On the Way to Language”, Harper San Francisco, pp. 57-108, 1982.

[43] M. Heidegger, "Schelling's Treatise on the essence of Human Freedom”, Ohio University Press, 1985.

[44] M. Heidegger, "Hegel's Concept of Experience", Harper \& Row, Publishers, San Francisco, 1989.

[45] M. Heidegger, "Modern science metaphysics and mathematics", in "Basic Writings", Routledge \& Kegan Paul. London, pp. 268-305, 1993a.

[46] M. Heidegger, "On the essence of truth", in "Basic Writings", Routledge \& Kegan Paul. London, pp. 115-138, 1993b.

[47] M. Heidegger, "The Way to Language", in "Basic Writings", Routledge \& Kegan Paul. London, pp. 397-426, 1993c

[48] H. López-Garay, "A Holistic Interpretive Concept of Systems Design”, Ph.D. thesis, University of Pennsylvania, Philadelphia, 1986.

[49] H. López-Garay and R.T. Suárez, "The holistic sense of prison phenomena in Venezuela: III. The unity of the research", Systemic Practice and Action Research, Vol. 12, No. 1, p. 115136, Plenum Press, New York and London, 1999.

[50] J.L. Lyotard, "The Postmodern Condition: A Report on Knowledge”, Manchester University Press, Manchester, 1984.

[51] A. MacIntyre, "After Virtue: a study in moral theory", Duckworth, Great Britain, 1981. 
[52] A. MacIntyre, “Whose Justice? Which Rationality?”, University of Notre Dame Press, Indiana, 1988.

[53] A. MacIntyre, "Three Rival Versions of Moral Enquiry", Duckworth. Great Britain, 1990.

[54] A. Ochoa-Arias, "An Interpretive-Systemic framework for the study of Community Organizations", Systemic Practice and Action Research, Vol.11, No. 5, 1998a.

[55] A. Ochoa-Arias, "Una aproximación crítica al papel de la organización comunitaria en el ámbito del Desarrollo Sostenible”, Revista Iberoamericana de Autogestión y Acción Comunal (Julio, 1998) pp. 53-63, 1998b.

[56] A. Ochoa-Arias, "Community Organizations in Venezuela: Toward the Disintegration of the Modern State and the Emergence of a "Community Organized" Society?", Systemic Practice and Action Research. Vol. 13 No. 2. Plenum Press, pp. 165-185, 2000.

[57] A. Ochoa-Arias, "Communautes et Epaces Publics dans le Pays du Sud", in Ghorra-Gobin (ed.) "Réinventer le sens de la Ville: Les espaces publics á l'heure globale”, L'Harmattan. Paris, pp. 47-57, ISBN 2-7475-0523-5, 2001.

[58] A. Ochoa-Arias, "An Interpretive Systemic exegesis of Community Action in Venezuela", in G. Midgley and A. OchoaArias, "Community Operational Research: OR and Systems Thinking for Community Development”, New York, Kluwer Academic Press, 2004a.

[59] A. Ochoa-Arias, "Organización Comunitaria y espacio público en sociedades periféricas a la modernidad", Boletín Antropológico, Año 22, No. 62, Septiembre-Diciembre 2004, pp. 351-367, 2004b.

[60] R. Rorty, "Philosophy and the Mirror of Nature", Blackwell. Oxford. UK, 1980.

[61] R.T. Suárez, "An inquiry into the historical meaning of The Fifth Discipline", Systemic Practice and Action Research, Vol. 11, No. 5, 1998, p. 483-502. Plenum Press. New York and London, 1998.

[62] R.T. Suárez, "The holistic sense of prison phenomena in Venezuela: II. Toward a profound unveiling of the "background", Systemic Practice and Action Research, Vol. 12, No. 1, p. 95-113. Plenum Press. New York and London, 1999a.
[63] R. T. Suárez, "El carácter problemático de la situación penitenciaria venezolana: hacia una solución de fondo", Frónesis, Vol. 6, No. 1, p. 85-115, Universidad del Zulia, Maracaibo, Venezuela, 1999b.

[64] R.T. Suárez, "Cárceles sin fin”, Consejo de Publicaciones de la Universidad de los Andes, Mérida, Venezuela, 2000.

[65] R. T. Suárez, "El sentido histórico del proyecto educativo de Lutero (I)”, Frónesis, Vol. 10, No. 3, p. 9-56, Universidad del Zulia, Maracaibo, Venezuela, 2003.

[66] R. T. Suárez, "El sentido histórico del proyecto educativo de Lutero (II)”, Frónesis, Vol. 11, No. 1, p. 41-81, Universidad del Zulia, Maracaibo, Venezuela, 2004.

[67] R. T. Suárez, "Esbozo de una historia-ontológica de la educación moderna y muestra del diseño de actividades pedagógicas para el $7^{\circ}$ y $8^{\circ}$ de educación básica. (Un aporte al Proyecto de Educación de la Sistemología Interpretativa)", [Tesis doctoral], Mérida, Venezuela, Universidad de Los Andes, 2005.

[68] Ch. Taylor, "Sources of the Self", Harvard University Press, Cambridge, Massachusetts, 1989.

[69] F. Varela, "De cuerpo presente", Barcelona, Editorial Gedisa, 1997.

[70] F. Varela, "La ciencia del ser. Las rutas de Francisco Varela", Valparaíso-Chile: Universidad de Valparaíso-Editorial, 2011.

[71] M. Villarreal, "La tecnocracia y el fracaso de la modernidad". En El poder de los expertos: Para comprender la tecnocracia", (Coordinadores: Haydée Ochoa y Alejandro M. Estévez). Universidad del Zulia. Venezuela., 2005.

[72] M.Villarreal, "La trayectoria de un enfoque de sistemas como fuente de sentido para la propuesta de un proyecto de educación para la Venezuela del siglo XXI", Revista Iztapalapa, No. 60, División de Ciencias Sociales y Humanísticas, Universidad Autónoma Metropolitana, México, D.F., 2006

[73] M. Villarreal, "Inicio en la Grecia Clásica de una historia ontológica y muestra del diseño de actividades pedagógicas para $1^{\circ}, 2^{\circ}, 3^{\circ}$ y $5^{\circ}$ grado de educación primaria (Un aporte al Proyecto de Educación de la Sistemología Interpretativa)", [Tesis doctoral]. Mérida, Venezuela: Universidad de Los Andes, 2007.

[74] L. Wittgenstein, "Philosophical Investigations", Basil Blackwell,
Oxford,1978. 